\title{
Neutrino flux predictions for galactic plerions
}

\author{
Dafne Guetta $^{\mathrm{a}, 1} \&$ Elena Amato ${ }^{\mathrm{a}, 2}$

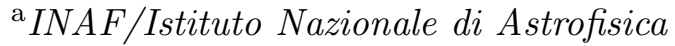 \\ Osservatorio astrofisico di Arcetri \\ Largo E. Fermi 5, I-50125 Firenze, Italy
}

\begin{abstract}
We consider a class of plerions that have been detected in the $\mathrm{TeV}$ range, and investigate the possibility that the emission is due to $\pi^{0}$ decay. From the TeV flux we derive what is the expected $\nu$ flux at Earth and find that this is likely to exceed the detection threshold of the upcoming $\mathrm{km}^{2}$ neutrino detectors.
\end{abstract}

Key words: High energy photons, Neutrinos, Supernova remnants PACS: : 95.85.Ry, 96.40.Tv, 97.60.Gb

\section{Introduction}

Plerions are supernova remnants (SNRs) with a filled morphology. These remnants are characterized by a center-brightened nebula often seen in the radio and X-ray wavelenghts and believed to be powered by an embedded pulsar. Typified by the Crab Nebula, they have non thermal spectra at all wavelengths. They have a flat power-law spectral index $\left(\alpha \sim 0-0.3, S_{\nu} \sim \nu^{-\alpha}\right)$ in the radio band and hard photon index $(\gamma \sim 2, \gamma=\alpha+1)$ in the X-ray band. Out of $\sim 220$ Galactic SNRs only about $10 \%$ are classified as plerions (or Crab-like SNRs) [10].

Plerion spectra are usually well interpreted from the radio to the X-ray band as synchrotron emission of a population of relativistic pairs continuously supplied by the central pulsar. At higher energies the Inverse Compton Scattering of the same electrons and positrons off either an internal or external target radiation can play a role. However it is not clear whether this latter process

1 E-mail: dafne@arcetri.astro.it

2 E-mail: amato@arcetri.astro.it

Preprint submitted to Elsevier Science 27 June 2018 
can be responsible for the emission recently observed from a few objects at $\mathrm{TeV}$ energies [16]. An alternative mechanism to produce $\mathrm{TeV}$ photons may be the decay of neutral pions produced through nuclear collisions of relativistic protons.

In this paper we investigate the consequences of a possible hadronic origin of these $\mathrm{TeV}$ detections. Following the line of a recent work by Alvarez-Muñiz \& Halzen [3], we compute the high energy neutrino flux at earth and find that the predicted fluxes may be detectable by large, $\mathrm{km}^{2}$ effective area, high energy neutrino telescopes, such as the planned south pole detector IceCube [12] or the Mediterranean sea detectors under construction (ANTARES, [4]; NESTOR, [14]) and planning (NEMO, [15]; see Ref. [11] for a recent review).

\section{TeV observations of plerions}

Four plerions have been so far detected at TeV energies, while upper limits exist for a few others. The objects for which the detection is at a high confidence level ( $\gtrsim 4 \sigma)$ are: the Crab Nebula [2], the Vela X SNR [20], the pulsar wind nebula around PSR1706-44 [13] and the radio nebula surrounding PSR150958 [16]. The VHE emission is unpulsed and therefore likely to be associated to the pulsar wind nebula rather than to the pulsar magnetosphere.

In Table 2 we report the list of pulsar wind bubbles which have been detected at $\mathrm{TeV}$ energies, supplied with the central pulsar luminosity and distance (Ref. [1] and references therein), and the observed TeV spectrum.

Two of the objects in the table deserve some comment. First of all, it should be noticed that the association between pulsar B1706-44 and the remnant G343.12.3 is questionable as discussed by Giacani et al.[9], and in the following we refer to the radio nebula detected by Frail et al.[6] as the remnant associated to this pulsar.

As to B1509-58, this pulsar is found in a very extended supernova remnant with a complex morphology. However a synchrotron nebula has been found with confidence surrounding the pulsar at X-ray frequencies $[17,18,5]$, although no pulsar wind bubble has been detected at radio frequencies [8]. Moreover the spectral index at $\mathrm{TeV}$ energies has not been determined with confidence [16]. In the following we use a value of 2.5 in analogy with the spectra of the

other objects and derive the normalization from the integrated photon flux measured by CANGAROO.

As we mentioned in the introduction, a possible mechanism to interpret the $\mathrm{TeV}$ fluxes is the Inverse Compton Scattering (ICS), on the ambient photon 
Table 1

Pulsar wind bubbles detected at TeV energies. The name of the pulsar and its associated remnant are reported in the first two columns. The pulsar bolometric luminosity and distance from Earth are in the third and fourth column respectively. In the last column the TeV spectrum as given in the references cited above is reported.

\begin{tabular}{|c|c|c|c|c|}
\hline 1 & 2 & 3 & 4 & 5 \\
\hline pulsar & SNR & $\begin{array}{c}L_{0} \\
10^{38} \mathrm{erg} / \mathrm{s}\end{array}$ & $\begin{array}{c}\mathrm{d} \\
\mathrm{kpc}\end{array}$ & $\begin{array}{c}\mathrm{dN} / \mathrm{dE} \\
10^{-11} \mathrm{~cm}^{-2} \mathrm{~s}^{-1} \mathrm{TeV}^{-1}\end{array}$ \\
\hline B0531+21 & Crab & 5 & 2 & $2.8(\mathrm{E} / \mathrm{TeV})^{-2.6}$ \\
\hline B0833-45 & Vela & 0.07 & .5 & $0.26(\mathrm{E} / 2 \mathrm{TeV})^{-2.4}$ \\
\hline B1706-44 & G343.1-2.3 ? & 0.034 & 1.8 & $0.23(\mathrm{E} / 1 \mathrm{TeV})^{-2.5}$ \\
\hline B1509-58 & MSH15-52 & 0.18 & 4.4 & $1.15(\mathrm{E} / 1 \mathrm{TeV})^{-2.5}$ \\
\hline
\end{tabular}

field, of the electrons responsible for the synchrotron emission at lower frequencies. The target radiation for ICS is given by the sum of two different contributions: the external photon field and the internal radiation produced by the source itself. A detailed discussion of the relative importance of the different contributions, within the context of the objects considered here, can be found in Ref. [1]. The conclusion is that the target photon density is expected to be dominated by the cosmic microwave background radiation (CMB) at 2.7 $\mathrm{K}$, corresponding to an energy density $w_{\mathrm{ph}}=0.26 \mathrm{eV} \mathrm{cm}^{-3}$.

When one considers the ICS and synchrotron emission in frequency ranges such that they are produced by the same electrons, the ratio of the synchrotron and ICS luminosities ( $L_{\mathrm{sync}}$ and $L_{\mathrm{ICS}}$, respectively) is equal to the ratio between the energy density of the magnetic and radiation fields. Since, as we mentioned above, the target radiation is mainly made of CMB photons, we can write, for the ratio between the ICS and synchrotron emission:

$$
\frac{L_{\mathrm{ICS}}}{L_{\mathrm{sync}}}=\frac{w_{\mathrm{CMB}}}{w_{\mathrm{B}}}
$$

where $w_{\mathrm{CMB}}$ and $w_{\mathrm{B}}$ indicate the $\mathrm{CMB}$ and magnetic field energy densities respectively. Eq. 1 can be used to estimate the magnetic field, once we specify the frequency bands in which $L_{\mathrm{ICS}}$ and $L_{\text {sync }}$ have to be taken.

The energy $E_{e}$ of an electron producing $\mathrm{TeV}$ photons by upscattering the $\mathrm{CMB}$ is given by:

$$
E_{e}[\mathrm{TeV}] \simeq 20\left(\frac{E_{\gamma}}{\mathrm{TeV}}\right)^{1 / 2}
$$

where $E_{\gamma}$ is the photon energy. Therefore the synchrotron photons emitted by 
the same electrons will have a typical energy of:

$$
\epsilon_{\text {sync }} \simeq 0.08 B_{-5} \mathrm{keV}
$$

where $B_{-5}$ is the nebular magnetic field in units of $10^{-5}$ Gauss. This value of the magnetic field strength is of order of that typically estimated for these nebulae assuming equipartition.

If the TeV flux is due to ICS on the CMB, we then find for the magnetic field strength:

$$
B_{I C}=3 \times 10^{-6}\left(\frac{L_{x}}{L_{\mathrm{TeV}}}\right)^{1 / 2} \text { Gauss }
$$

where $L_{\mathrm{TeV}}$ is the luminosity at $\mathrm{TeV}$ energies obtained integrating the $\mathrm{TeV}$ spectrum given in Table 2 above $1 \mathrm{TeV}$.

In Table 2 we compare the value of $B_{I C}$ with what can be found assuming equipartition $B_{\text {eq. }}$. For the first three remnants we have computed the equipartition magnetic field based on the radio data while in the case of the nebula surrounding PSR B1509-58 we have used the luminosity and size at X-ray frequencies, as given in Ref. [17].

Table 2

Values of the parameters used to estimate $B_{\mathrm{eq}}$ and $B_{\mathrm{IC}}$. The computed fields are reported in columns 6 and 8 respectively. In the second column we report the radio luminosity integrated over the frequency band specified in the adjacent column. The spectral index and the extension of the nebula at radio frequencies are reported in columns 4 and 5 respectively. The numbers we report for Crab and Vela are taken from the SNR Catalogue [10], while those for the nebulae associated to B1706-44 and B1509-58 are from the references given in the text. Finally, the X-ray luminosities given in column 7 are taken from [1].

\begin{tabular}{|c|c|c|c|c|c|c|c|}
\hline 1 & 2 & 3 & 4 & 5 & 6 & 7 & 8 \\
\hline pulsar & $\begin{array}{c}L_{R} \\
10^{35} \mathrm{erg} / \mathrm{s}\end{array}$ & $\begin{array}{c}\text { Frequency band } \\
\mathrm{GHz}\end{array}$ & $\begin{array}{c}\text { spectral } \\
\text { index }\end{array}$ & $\begin{array}{c}R_{N} \\
\mathrm{pc}\end{array}$ & $\begin{array}{c}\mathbf{B}_{\text {eq }} \\
\mu G\end{array}$ & $\begin{array}{c}L_{X} \\
10^{35} \mathrm{erg} / \mathrm{s}\end{array}$ & $\begin{array}{c}\mathbf{B}_{\text {IC }} \\
\mu G\end{array}$ \\
\hline B0531+21 & 1.7 & $10^{-2}-10^{2}$ & 0.3 & 1.5 & $\mathbf{3 0 0}$ & 150 & $\mathbf{2 3 0}$ \\
\hline B0833-45 & 0.04 & $10^{-2}-10^{2}$ & 0.3 & 0.2 & $\mathbf{6 5 0}$ & 0.12 & $\mathbf{6 4}$ \\
\hline B1706-44 & $7.6 \times 10^{-5}$ & $10^{-2}-10^{2}$ & 0.3 & 1.3 & $\mathbf{2 0}$ & 0.01 & $\mathbf{5}$ \\
\hline B1509-58 & - & - & - & 7 & $\mathbf{5}$ & 0.6 & $\mathbf{5}$ \\
\hline
\end{tabular}

It is apparent from the table that while for two of our four sources the ICS and the equipartition values of the field are very close, in the case of B1706-44 
and Vela a noticeable discrepancy (a factor of 4 and 10 respectively) is found. For these two objects the possibility that the $\mathrm{TeV}$ emission is due to hadronic processes is particularly appealing. If this is actually the case, then we expect a neutrino flux from these sources that we compute in the next section.

\section{Neutrino events}

A way to disentangle electromagnetic and hadronic sources of high energy $\gamma$-ray emission observationally is to look for the neutrino signals. Relativistic protons may produce $\mathrm{TeV} \gamma$-rays either by photo-meson production or inelastic nuclear collisions. The relative importance of the two processes depends on the target density of radiation and matter in the source. The main difference, as far as their outcome is concerned, is in the fraction of energy that goes into charged pions compared to neutral ones. This translates in a different ratio between the total neutrino and photon energy flux. In the case of plerions the most likely process at work is p-p scattering, as can be readily seen by comparing the rates of photomeson production and p-p scattering estimated below.

For photo-meson production the target for high energy protons is the plerion emission. The fractional energy loss rate of a proton with energy $E_{p}\left(=\Gamma m_{p} c^{2}\right)$ due to pion production results in (Ref. [19]):

$$
\begin{aligned}
t_{p \gamma}^{-1}\left(E_{p}\right) & \simeq \frac{2^{p+1}}{p+2} \sigma_{\text {peak }} \xi_{\text {peak }} \frac{\Delta \epsilon}{\epsilon_{\text {peak }}}\left(\frac{\mathrm{d}}{\mathrm{R}_{N}}\right)^{2} \frac{F_{\nu}\left(\nu_{\mathrm{p}}\right)}{4 \pi h} \\
& \simeq 2.5 \times 10^{-16} \frac{2^{p+1}}{p+2}\left(\frac{\mathrm{d}_{\mathrm{kpc}}}{\mathrm{R}_{\mathrm{pc}}}\right)^{2} F_{\nu_{\mathrm{p}}}[\mathrm{mJy}] \mathrm{yr}^{-1}
\end{aligned}
$$

Here we have treated the plerion as homogeneous and used the fact that the photon spectrum is a power law, $F_{\nu} \propto \nu^{-p}$. We have also made the approximation that the main contribution to pion production comes from photon energies $\epsilon_{\gamma} \approx \epsilon_{\mathrm{peak}}=0.3 \mathrm{GeV}$, where the p- $\gamma$ cross section peaks due to the $\Delta$ resonance. The numerical values are obtained using: $\sigma_{\text {peak }}=5 \times 10^{-28} \mathrm{~cm}^{2}$, $\xi_{\text {peak }}=0.2, \Delta \epsilon=0.2 \mathrm{GeV}, \nu_{\mathrm{p}}=\epsilon_{\text {peak }} /(\Gamma h)$ and $\beta_{p} \simeq 1$. Finally we have scaled the nebular radius and distance to the typical values of $1 \mathrm{pc}$ and $1 \mathrm{kpc}$, respectively $\left(\mathrm{R}_{\mathrm{pc}}=\mathrm{R}_{N} / \mathrm{pc}\right.$ and $\left.\mathrm{d}_{\mathrm{kpc}}=\mathrm{d} / \mathrm{kpc}\right)$, and expressed the nebular synchrotron radiation flux in units of $\mathrm{mJy}$.

The energy loss-rate of a relativistic proton due to inelastic nuclear collisions 
can be estimated as

$$
t_{p p}^{-1} \approx \zeta n_{t} \sigma_{0} c \approx \zeta \frac{\mathrm{M}_{N}}{m_{p}} \frac{3}{4 \pi \mathrm{R}_{N}^{3}} \sigma_{0} c,
$$

where $n_{t}$ is the target density, which we have expressed in terms of the nebular radius $R_{N}$ and content of thermal material $M_{N}$. Introducing in Eq. 6 the numerical values of the cross section for p-p scattering, $\sigma_{0}=5 \times 10^{-26} \mathrm{~cm}^{2}$, and of the average fraction of energy lost by the proton, $\zeta \simeq 20 \%$, we obtain:

$$
t_{p p}^{-1} \approx 10^{-7} \frac{M_{\mathrm{N} \odot}}{\mathrm{R}_{\mathrm{pc}}^{3}} \mathrm{yr}^{-1}
$$

where $M_{\mathrm{N} \odot}=M_{\mathrm{N}} / M_{\odot}$. From the comparison between Eq.7 and Eq.5, it is apparent that nuclear collisions are by far the most likely mechanism for pion production in plerions.

From this follows that the relation between the neutrino and photon flux is:

$$
\int_{E_{\nu}^{\min }}^{E_{\nu}^{\max }} E_{\nu} \frac{d N_{\nu}}{d E_{\nu}} d E_{\nu}=\int_{E_{\gamma}^{\min }}^{E_{\gamma}^{\max }} E_{\gamma} \frac{d N_{\gamma}}{d E_{\gamma}} d E_{\gamma}
$$

where $E_{\gamma}^{\min }\left(E_{\gamma}^{\max }\right)$ is the minimum (maximum) energy of the p-p produced photons and $E_{\nu}^{\min } \approx(1 / 2) E_{\gamma}^{\min }$ and $E_{\nu}^{\max } \approx(1 / 2) E_{\gamma}^{\max }$ are the corresponding minimum and maximum neutrino energies.

We estimate the neutrino flux in the energy range 1-100 $\mathrm{TeV}$, which is the range in which the future $\mathrm{km}^{2}$ neutrino detectors will operate. Therefore we are concerned with photon energies above $2 \mathrm{TeV}$.

In Table 3 we report the total number of neutrino events expected in a yr of operation of a $\mathrm{km}^{2}$ detector. This is obtained directly from:

$$
N_{\mu}=4 A_{\text {eff }} \times T \times \int_{1 \mathrm{TeV}}^{100 \mathrm{TeV}} \frac{d N_{\gamma}}{d E_{\gamma}}\left(2 E_{\nu}\right) P_{\nu \mu}\left(E_{\nu}\right) d E_{\nu},
$$

where $P_{\nu \mu}=1.3 \cdot 10^{-6} E_{\nu, \mathrm{TeV}}[7]$ is the detection probability for neutrinos with $E_{\nu} \gtrsim 1 \mathrm{TeV}, T$ is the observation time and $A_{\text {eff }}$ is the effective area.

The number of atmospheric neutrino events collected in a $\mathrm{km}^{2}$ detector during $1 \mathrm{yr}$ is of order 1 . This is estimated assuming a background neutrino spectrum $\phi_{\nu, \mathrm{bkg}} \sim 10^{-7} E_{\nu, \mathrm{TeV}}^{-2.5} \mathrm{~cm}^{-2} \mathrm{~s}^{-1} \mathrm{sr}^{-1}$ for $E_{\nu}>1 \mathrm{TeV}$, and a detector angular resolution of $0.3^{\circ}$ like NEMO [15]. 
Table 3

Predicted number of muon events, $N_{\mu}$, in a $\mathrm{km}^{2}$ detector, using Eq.9 for the plerions considered in the paper. One year of integration time is assumed.

\begin{tabular}{|c|c|}
\hline pulsar & $N_{\mu}$ \\
\hline $\mathrm{B} 0531+21$ & 11.8 \\
\hline $\mathrm{B} 0833-45$ & 9.0 \\
\hline $\mathrm{B} 1706-44$ & 1.2 \\
\hline $\mathrm{B} 1509-58$ & 6.0 \\
\hline
\end{tabular}

Our calculation suggests that the neutrino signal from three of the plerions considered here maybe identified above the atmospheric neutrino background by the next generation underwater (ice) neutrino telescopes.

\section{Discussion}

We have investigated the implications of a possible hadronic origin of the high energy emission from the plerions which have been detected by CANGAROO at $\mathrm{TeV}$ energies. The alternative explanation for this emission is based on ICS. From the comparison of the implied magnetic field strength under this latter assumption with the equipartition value, we have found that the ICS origin of $\mathrm{TeV}$ photons poses some difficulties for at least two of our four sources, namely Vela and the remnant surrounding B1706-44.

We have computed the implied neutrino flux under the assumption that the emission seen by CANGAROO is due to p-p scattering. We conclude that, for three of these objects, this hypothesis can be confirmed or disproven with confidence, given that the upcoming neutrino telescopes will be operated for a few years.

The positive detection of neutrinos with $E_{\nu} \gtrsim \mathrm{TeV}$ would not only provide the final answer to the origin of the detected VHE $\gamma$-rays but also confirm the hypothesis that protons can be accelerated upto at least tens of $\mathrm{TeV}$ in these objects.

\section{Acknowledgments}

We thank the referee for his suggestions. We aknowledge M. Salvati and F. Pacini for useful conversations. 


\section{References}

[1] Aharonian F.A., Atoyan A.M., \& Kifune T., 1997, MNRAS, 291, 162

[2] Aharonian F.A., et al., 2000, ApJ, 539, 317

[3] Alvarez-Muñiz J. \& Halzen F., astro-ph/0205408

[4] ANTARES Proposal, 1997, astro-ph/9707136

[5] Brazier K.T.S. \& Becker W., 1997, MNRAS, 284, 335

[6] Frail D.A., Goss W.M., \& Whiteoak J.B.Z., 1994, ApJ, 437, 781

[7] Gaisser T.K., Halzen F. \& Stanev T., 1995, Phys. Rep., 258, 173

[8] Gaensler B.M., et al., 1999, MNRAS, 305, 724

[9] Giacani E.B., et al., 2001, ApJ, 121, 3133

[10] Green D.A., 2001, 'A catalogue of Galactic Supernova Remnants', (http://www.mrao.cam.ac.uk/surveys/snrs )

[11] Halzen F., 2001, in Intl. Symp on High Energy Gamma Ray Astronomy, Heidelberg, June 2000 (astro-ph/0103195)

[12] IceCube Proposal, (http://www.ssec.wisc.edu/a3ri/icecube/overview/ original_nsf_proposal)

[13] Kifune T., et al., 1995, ApJ, 438, 91

[14] Monteleoni B. for the NESTOR Collaboration, 1996, Proceedings of the XVII International Conference on Neutrino

[15] Riccobene G. for the NEMO Collaboration, to appear on the proceedings of the "Workshop on methodical aspects of underwater/ice neutrino telescopes", Hamburg, 15-16 August 2001

[16] Sako T., et al.., 2000, ApJ, 537, 422

[17] Seward F.D., et al., 1984, ApJ, 281, 650

[18] Tamura K., et al., 1996, PASJ, 48, L33

[19] Waxman E. \& Bahcall J.N., 1997, Phys. Rev. Lett., 78, 2292

[20] Yoshikoshi T., et al., 1997, ApJ, 487, L65 